\title{
Experimental Investigation of Inter-Modal Cross-Gain Modulation and Transient Effects in a Two Mode Group Erbium Doped Fiber Amplifier
}

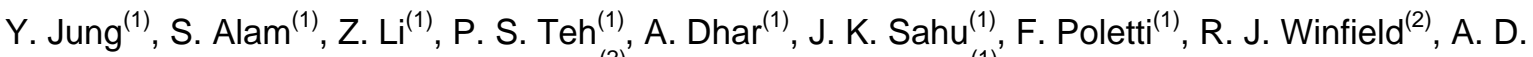 \\ Ellis $^{(2)}$, and D. J. Richardson ${ }^{(1)}$
}

(1) Optoelectronics Research Centre, University of Southampton, Southampton, SO17 1BJ, UK

(2) Tyndall National Institute, University of College Cork, Cork, Ireland ymj@orc.soton.ac.uk

\begin{abstract}
We report what we believe to be the first experimental study of inter-modal cross-gain modulation and associated transient effects as different spatial modes and wavelength channels are added and dropped within a two-mode amplifier for SDM transmission.
\end{abstract}

\section{Introduction}

Spatial division multiplexing (SDM) technology $y^{1,2}$ is currently being explored as a potential way forward to meet the ever-increasing global demands for transmission bandwidth, providing an additional degree of freedom to up-scale fiber capacity. As a consequence a range of modeselective components such as phase plates, long period fiber gratings, and spatial light modulators are being developed to help realize mode multiplexers/demultiplexers to enable transmission in few mode fibers, whilst in-line few mode amplifiers have already been demonstrated and employed in transmission experiments over an amplified two-span fiber link $^{2}$. In our recent work $^{3}$ we successfully demonstrated a two-mode group erbium-doped fiber amplifier (TM-EDFA) for SDM applications capable of simultaneously amplifying six separate spatial modes (including all polarizations and degeneracies). In our initial experiments we showed the amplifier to be capable of providing simultaneous modal gains of $>20 \mathrm{~dB}$ for different pair-wise combinations of spatial and polarization modes with modest values (2-3dB) of differential modal gain. One of the most interesting, but to date uninvestigated, aspects of TM-EDFA operation is the question of cross-gain modulation and the associated gain dynamics (or transient response) between and indeed within spatial mode groups as the number of channels within the device is changed. Such effects, already extensively studied both theoretically and experimentally for single mode EDFAs $s^{4-5}$, will certainly represent an important issue to allow practical application of the SDM technology within robustly reconfigurable optical networks.

In this paper, we investigate for the first time a study of the gain dynamics within a TM-EDFA investigating both power excursions due to cross gain modulation and transient times for the two LP mode groups $\left(\mathrm{LP}_{01}\right.$ and $\left.\mathrm{LP}_{11}\right)$ supported.

\section{Experimental setup}

Our experimental setup for the investigation of the transient effects in a TM-EDFA is shown in Fig. 1. At the mode multiplexer, three channels at different wavelengths (two $\mathrm{LP}_{01}$ and one $\mathrm{LP}_{11}$ ) were multiplexed using free space optics. A binary phase plate $(0, \pi)$ was used to generate the $\mathrm{LP}_{11}$ mode from an incident $\mathrm{LP}_{01}$ mode and all three beams were combined using two $3 \mathrm{~dB}$

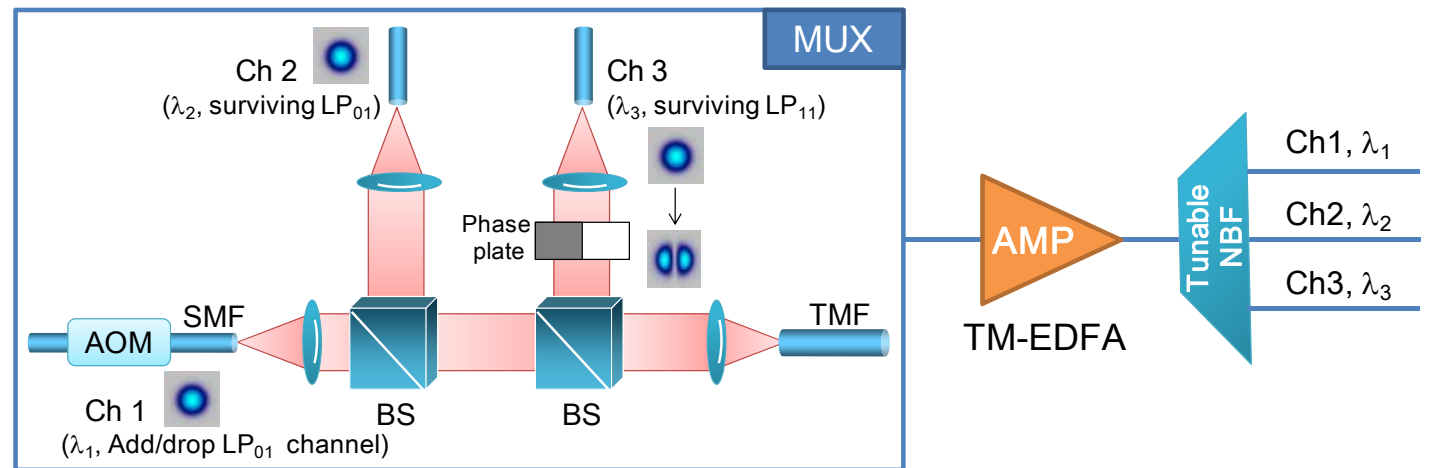

Fig. 1: Experimental setup for investigating transient effects in a TM-EDFA. BS: beam splitter, AOM: acoustooptic modulators, SMF: single mode fiber, TMF: two mode fiber, Ch1: channel 1, NBF: narrow bandwidth filter. 
polarization insensitive beam splitters and coupled into a passive TM-fiber via suitable focusing optics. An acousto-optic modulator (AOM) was placed in one of the $\mathrm{LP}_{01}$ lines, which we refer to as Ch1, and was used to generate a square wave with $50 \%$ duty cycle in order to allow us to simulate the impact of adding/dropping one (or multiple) spatial channels. Ch2 ( $\left.\mathrm{LP}_{01}\right)$ and $\mathrm{Ch} 3\left(\mathrm{LP}_{11}\right)$ were used as "surviving channels" to investigate the mode dependent gain saturation and associated transient effects. The TM-fiber output from the multiplexer was spliced to a $5 \mathrm{~m}$ long specially designed two-mode erbium doped fiber (TMEDF) with a tailored central dip in both the refractive index and $\mathrm{Er}^{3+}$-ion distribution which serve to reduce differential modal gain. As reported in our previous study ${ }^{3}$, the TM-EDFA was designed to amplify two transverse mode groups $\left(\mathrm{LP}_{01}, \mathrm{LP}_{11}\right)$ simultaneously. A 980nm fiber pigtailed single mode diode laser was used to pump the active fiber in a counter-propagating configuration and its output was free-space coupled into the TM-EDF using two dichroic mirrors. An offset pump launch scheme which preferentially launches the pump light into higher order modes was adopted in order to further minimize the gain differential between the $L P_{01}$ and $\mathrm{LP}_{11}$ modes. For ease of demultiplexing the individual channels at the output of the TMEDFA, the wavelengths of Ch1, Ch2, and Ch3 were chosen to be $1548 \mathrm{~nm}, 1553 \mathrm{~nm}$, and $1558 \mathrm{~nm}$ respectively. A tunable narrow bandpass filter (NBF, $\Delta \lambda=2 \mathrm{~nm}$ ) was then used at the output of the amplifier to separate the individual channels. The temporal response of each channel was recorded using $1.2 \mathrm{GHz}$ InGaAs photodiodes (DET01CFC, Thorlabs) and a digital oscilloscope (TDS5032B, Tektronix). Unless otherwise stated the input power for each channel was kept constant at $6 \mathrm{dBm}$ (which we take to be representative of the power of a single "data stream") and the pump power was fixed at $23 \mathrm{dBm}$.

\section{Transient response of a TM-EDFA}

Fig. 2 shows the normalized input and output signal channels as one of the channels is modulated. In Fig. 2a the add/drop input channel Ch1 has a power of $0 \mathrm{dBm}$ (equivalent in power terms to 4 data streams each of $-6 \mathrm{dBm}$ power) and was modulated using the AOM with a $50 \%$ duty cycle and a frequency of $10 \mathrm{~Hz}$. The input of the surviving channels was kept constant (Ch2 $=-6 \mathrm{dBm}, \mathrm{Ch} 3=-6 \mathrm{dBm}$ equivalent to the power of 1 data stream of $-6 \mathrm{dBm}$ power). The temporal measurements of the output signal channels are shown in Fig. 2b-d. All channels
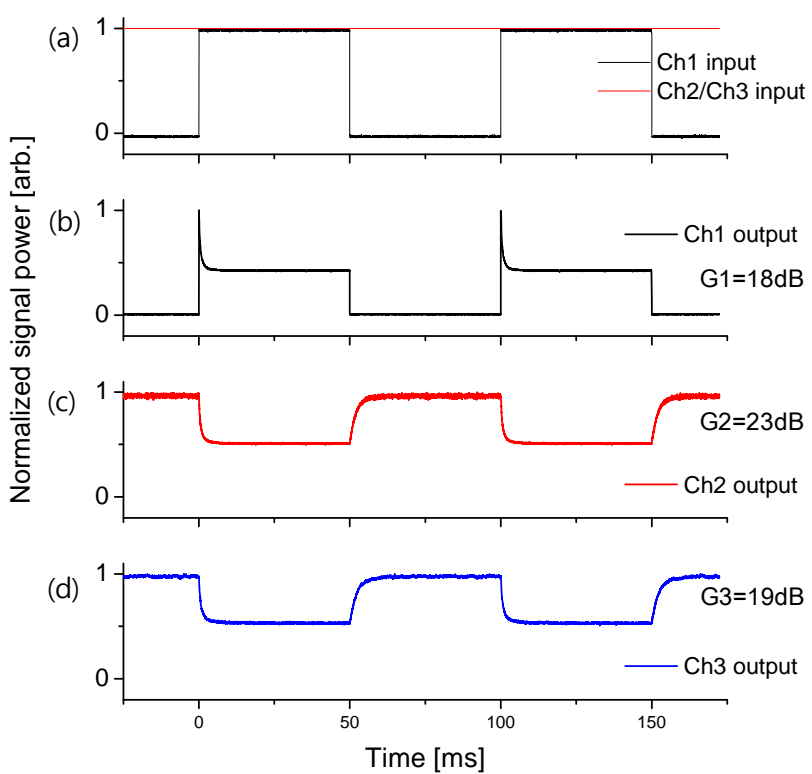

Fig. 2: Normalized (a) input and (b-d) output signal channels in the event of modulation of Ch1.

experience significant signal power excursions (defined as the ratio between the maximum and minimum channel power in the absence and presence respectively of the Ch1 signal) as a result of cross gain saturation in the TM-EDFA. During the channel-add event (presence of Ch1), the surviving channels experience a drop in output power due to the increased competition for gain from the available population inversion from Ch1. Conversely, during the channel-drop event, the surviving channels experience a sudden increase in inversion due to the disappearance of Ch1 and the original output power levels are restored. It is to be noted that the transient responses of two different spatial modes $\left(\mathrm{LP}_{01}\right.$ in $\mathrm{Ch} 2$ and $\mathrm{LP}_{11}$ in $\left.\mathrm{Ch} 3\right)$ are very similar.

To simulate the effects of adding/dropping multiple data streams the power of Ch1 was varied from $-6 \mathrm{dBm}$ (1 data stream) to $0 \mathrm{dBm}$ (4 data streams). The results are shown in Fig. 3a, where the power excursion for both $\mathrm{Ch} 2$ and Ch3 increases with Ch1 input signal power. For the surviving $\mathrm{LP}_{01}$ channel (Ch2), the power excursion increases from $1 \mathrm{~dB}$ for the single data stream drop case to $2.8 \mathrm{~dB}$ for the 4 stream case, whereas the increase is from $1 \mathrm{~dB}$ to $2.6 \mathrm{~dB}$ for the surviving $\mathrm{LP}_{11}$ channel (Ch3). The $0.2 \mathrm{~dB}$ maximum power excursion difference between two spatial modes for a Ch1 power of $0 \mathrm{dBm}$ is due to the different spatial overlap between the $\mathrm{LP}_{01}$ and $\mathrm{LP}_{11}$ modes. One would expect $\mathrm{Ch} 2$ on the same $\mathrm{LP}_{01}$ spatial mode as modulated Ch1 to see a greater power excursion than $\mathrm{Ch} 3$ on the $\mathrm{LP}_{11}$ mode since it sees exactly the same spatial distribution of ions as Ch1. We would expect this difference to increase with increasing number of add/drop data streams, or when 
$-{ }^{-5}$.pdf

(a)
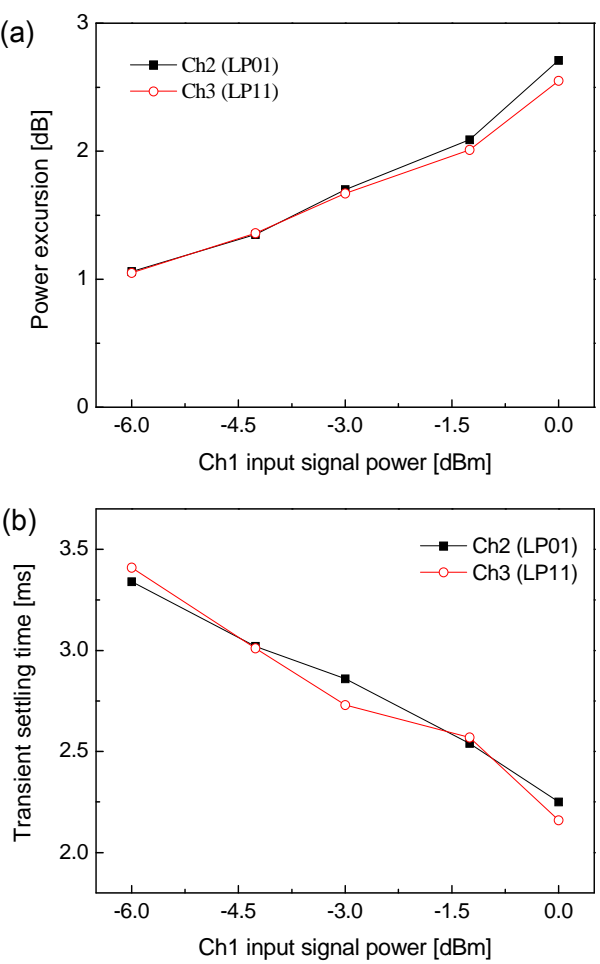

Fig. 3: Transient responses (a: power excursion, b: transient time) of surviving channels according to Ch1 input signal power.

operating the system more strongly in the large signal (saturated) regime.

From Fig. 2, we can define the transient settling time (rise or fall time) as the time taken for the power excursion to go from $10 \%$ to $90 \%$ of the maximum steady state power excursion. In Fig. $3 \mathrm{~b}$, we plot the measured transient settling time for both spatial modes. Both exhibit almost identical behavior with a settling time decreasing from $3.4 \mathrm{~ms}$ to $2.2 \mathrm{~ms}$ when increasing the Ch1 input signal power from $-6 \mathrm{dBm}$ to $0 \mathrm{dBm}$. Generally, the transient settling time is dependent on the level of saturation of the amplifier and in particular to be shorter for higher pump or signal power. Consequently the transient response becomes faster as the Ch1 input signal power increases. To check the pump power dependence, we changed the pump power from $20.2 \mathrm{dBm}$ to $26.2 \mathrm{dBm}$ with a constant signal input power of Ch1 $(0 \mathrm{dBm})$. The trend was essentially the same as that seen for the Ch1 input signal power changes in Fig. 3b, and we confirmed that even higher pump power provides an even faster transient settling time.

To examine the dependence of the power excursion on modulation frequency the modulation frequency of the AOM was gradually increased from $10 \mathrm{~Hz}$ to $5 \mathrm{kHz}$ with a constant amplifier input signal power (Ch1=0dBm, Ch2=$6 \mathrm{dBm}, \mathrm{Ch} 3=-6 \mathrm{dBm})$ and pump power (23.1dBm). As shown in Fig. 4a, the total power excursion decreases as the modulation frequency is increased. At frequencies higher than $5 \mathrm{kHz}$ the (a)

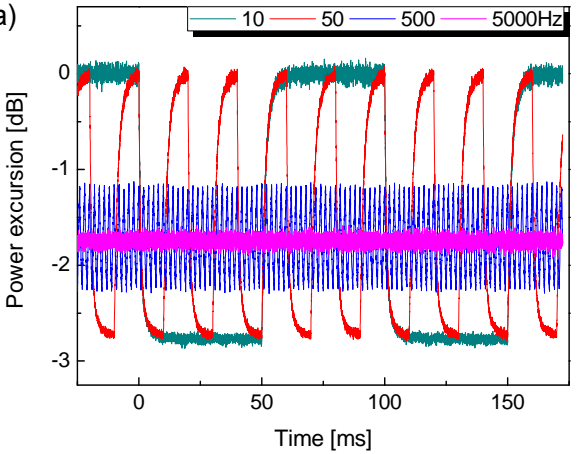

(b)

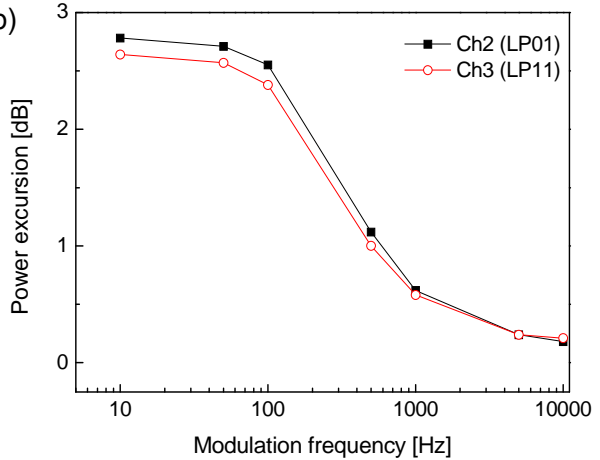

Fig. 4: Power excursion as a function of the modulation frequency.

surviving channels cannot follow the fast modulation and only a steady-state gain compression appears due to the slow response of fiber amplifier's population inversion, which has an excited state lifetime of $\sim 10 \mathrm{~ms}$. As shown in Fig. 4b, the total power excursion for the $\mathrm{LP}_{01}$ and $L \mathrm{P}_{11}$ modes at $10 \mathrm{~Hz}$ was $2.8 \mathrm{~dB}$ and $2.6 \mathrm{~dB}$, respectively, which decreased to less than $0.2 \mathrm{~dB}$ at modulation frequencies of higher than $5 \mathrm{kHz}$.

\section{Conclusions}

We have investigated inter-modal cross gain and associated transient effects in a TM-EDFA for the first time. Gain measurements for the $\mathrm{LP}_{01}$ and $\mathrm{LP}_{11}$ spatial modes were made as different effective numbers of data streams were added or dropped. Our results indicate that all modes experience roughly similar responses under a range of different add/drop conditions, although some evidence of mode dependent sensitivity was observed in experiments operating at higher levels of amplifier saturation.

\section{Acknowledgements}

This work was supported by the European Communities $7^{\text {th }}$ Framework Programme under grant agreement 228033 (MODE-GAP).

\section{References}

[1] R. Ryf et al., J. Light. Tech. 30, 521 (2012).

[2] N. Bai et al., Opt. Express 20, 2668 (2012).

[3] Y. Jung et al., Opt. Express 19, B952 (2011).

[4] C.R.Giles et al., Opt. Lett. 14, 880 (1989).

[5] Y. Sun et al., Bell Labs Tech. J., 187 (1999). 